\title{
ORIGINAL
}

\section{RESULTADOS DEL PROGRAMA DE DETECCIÓN PRECOZ DE CÁNCER COLORRECTAL EN CANTABRIA DURANTE EL PERIODO NOVIEMBRE DE 2008 A MARZO DE 2010}

\author{
Verónica Brugos-Llamazares (1), Álvaro González de Aledo Linos (1), Javier Vada-Sánchez (1) y \\ Álvaro Terán-Lantarón (2) \\ (1) Sección de Promoción y Educación para la Salud, Dirección General de Salud Pública. Consejería de Sanidad. \\ Gobierno de Cantabria. Santander. Cantabria. España. \\ (2) Servicio de Digestivo, Hospital Universitario Marqués de Valdecilla. Santander. Cantabria. España.
}

\section{RESUMEN}

Fundamento: Por su elevada incidencia y mortalidad el cáncer colorrectal (CCR) se considera un importante problema de Salud Pública. Los programas de cribado están adquiriendo cada vez mayor relevancia en su abordaje dada la importancia del diagnóstico precoz en la disminución de la mortalidad por esta causa. El objetivo de este trabajo es presentar el desarrollo y resultados del programa de cribado poblacional de CCR en Cantabria.

Métodos: Estudio descriptivo del programa de cribado de carácter bienal diseñado para la población de 55 a 69 años a partir de la base de datos de la Tarjeta Sanitaria ( 92.605 personas). La determinación de sangre oculta en heces $(\mathrm{SOH})$ por método inmunoquímico cualitativo es la prueba de cribado, empleándose la colonoscopia óptica como confirmación. La población recibe una carta de invitación, debiéndose citar en su Centro de Salud si aceptan participar. Tras aplicar los criterios de exclusión, las personas con resultado positivo en el test son citadas para colonoscopia. Aquellos en los que se detecta un carcinoma invasivo son remitidos directamente al Servicio de digestivo.

Resultados: En el periodo de estudio estaban cubiertas por e programa 35.005 personas $(37,8 \%$ de la población diana). De las invitadas (7.406) participaron $2.384(32,1 \%)$. Fueron detectados un carcinoma «in situ», 2 invasivos y 43 adenomas de alto riesgo.

Conclusión: Por su integración en Atención Primaria, ausencia de aparataje, fácil uso y rápida realización, el test de detección de SOH inmunoquímico cualitativo facilita la extensión del cribado, con resultados similares a los de los test inmunoquímicos cuantitativos.

Palabras clave: Cáncer colorrectal. Programa de cribado. Sangre oculta.

Correspondencia:

Verónica Brugos-Llamazares.

C/Juan de Herrera 4, $3^{\circ}$ izq.

39002 Santander.

Correo electrónico: brullaver@hotmail.com

\section{ABSTRACT \\ Results of the Screening Program for Colorectal Cancer Screening in Cantabria, Spain, during the Period November 2008-March 2010}

Background: The colorectal cancer (CCR) is a Public Health problem because of its high incidence and mortality. Screening programs are important for early detection of CCR. An early detection of this illness could decrease its mortality. The objectives of our study are to present the colorectal cancer screening program in Cantabria and its main results.

Methods: Descriptive study of a two-year screening program for population in the $55-69$ years age range, according to the Cantabria Health Service data base $(92,605$ people). The screening test used a qualitative immunochemical method in search of faecal occulted blood (FOB), complemented by optical colonoscopy confirmation. Eligible people were invited to participate in the screening program by means of personal letter and, in case of acceptance, they were dated in their primary Health Center. After application of exclusion criteria, selected people with a positive result test were submitted to optical colonoscopy. Those in which an invasive cancer was detected were directly addressed to the Digestive Service.

Results: The program was offered to 35,005 (37.8\%) of the eligible population of Cantabria at the end of study period. 2,384 persons participated $(32.1 \%)$. The main colonoscopy findings were 1 «in situ» cancer, 2 invasive cancer and 43 high-risk adenomas.

Conclusion: Due to its integration into primary care, lack of apparatus, ease use and fast implementation, qualitative immunochemical test FOBT facilitates the extension of screening, with similar results to those of quantitative immunochemical test.

Key words: Colorectal cancer. Screening . Occult blood

Financiación: «Sin financiación».

Conflictos de interés: Los autores declaran no tener ningún conflicto de intereses. 


\section{INTRODUCCIÓN}

En el año 2002 el cáncer colorrectal (CCR) era el segundo cáncer más común ${ }^{1}$ en el mundo, teniendo en cuenta su prevalencia total (en hombres y mujeres). En el año 2006 era la segunda causa de muerte por cáncer en Europa $^{2}$, por detrás del de pulmón, el segundo cáncer más frecuentemente diagnosticado en mujeres después del cáncer de mama y el tercero en hombres por detrás de los de próstata y pulmón ${ }^{2}$.

España ocupa una situación intermedia en Europa respecto a la incidencia y mortalidad por CCR. Se estima que el número de casos nuevos anuales diagnosticados en ambos sexos está en torno a los 22.000 , contabilizándose 13.075 defunciones anuales por esta causa $^{3}$ (7.585 en hombres ${ }^{3}$ y 5.490 en mujeres $\left.^{3}\right)$. Es por ello que podemos decir que el CCR es un importante problema de Salud Pública.

Se han propuesto distintas estrategias para abordarlo (prevención primaria, secundaria, mejoras terapéuticas, etc...). Debido a los avances habidos en los últimos años en el diagnóstico y tratamiento del CCR y a la importancia que tiene para la disminución de la mortalidad el diagnóstico precoz, los programas de cribado están adquiriendo cada vez mayor relevancia.

En el momento actual de todas las posibles pruebas diagnósticas disponibles para la realización de cribados poblacionales de $\mathrm{CCR}$ (sangre oculta en heces - $\mathrm{SOH}-$, exámenes radiológicos y endoscópicos) la detección de $\mathrm{SOH}$ se presenta como la más eficaz, disminuyendo la mortalidad por esta causa $^{4-7}$ y también la incidencia ${ }^{8}$. Esto, unido a su bajo coste, fácil realización y escasos eventos adversos, ha hecho que sea la prueba de cribado más seleccionada en Europa.

La recomendación de distintos organismos para la prevención del CCR es llevar a cabo cribados poblacionales en personas con riesgo medio para desarrollar $\mathrm{CCR}$, esto es aquéllas que tienen entre 50 y 74 años sin otros factores de mayor riesgo, mediante el test de $\mathrm{SOH}$ con carácter anual o bienal ${ }^{9-13}$.

Cantabria cuenta con una población de 591. 886 personas, de las cuales el $28 \%$ (164.596) tienen entre 50 y 74 años ${ }^{14}$. Para el periodo 1997-2003 la tasa bruta de incidencia de CCR en ambos sexos fue de 58,34 casos por 100.000 habitantes ${ }^{15}$. Para el periodo 1.997-2.006 la tasa bruta de mortalidad por este cáncer fue de 29,64/100.000 habitantes.

En el año 2007 desde la Consejería de Sanidad se adoptó la decisión de implementar el cribado del CCR de forma universal aprobándose el plan diseñado para el mismo ${ }^{15}$ en el año 2008 e iniciándose en noviembre de ese mismo año una fase piloto en 4 Centros de Salud con la decisión tomada de tenerlo extendido en toda la población en un corto periodo de tiempo (el año 2010). Se diseñó un programa de cribado de carácter bienal para la población susceptible de Cantabria. A partir de enero de 2010 se inició su progresiva extensión a toda la población.

El objetivo de este trabajo es presentar el desarrollo y los resultados alcanzados por el programa desde su inicio en noviembre de 2008 hasta marzo de 2010.

\section{SUJETOS Y MÉTODOS}

Diseño. Estudio descriptivo del programa de cribado poblacional de CCR para el periodo del 1 de noviembre de 2008 al 31 de marzo de 2010 (17 meses).

Población diana. Personas de 55 a 69 años de la base de datos de la Tarjeta Sanitaria. Los criterios de exclusión fueron los siguientes:

a) Temporales: enfermedad o invalidez grave para la que se prevea recupera- 
ción; examen endoscópico (sigmoidoscopia o colonoscopia) en los 5 años previos (10 si la colonoscopia fue completa); menstruación, hematuria o sangrado anal visible; antecedentes personales de patología de colon susceptible de seguimiento colonoscópico (colitis ulcerosa, enfermedad de Crohn) y que hayan realizado la colonoscopia en los últimos 3 años.

b) Definitivos: antecedentes personales de CCR; antecedentes familiares de CCR y/o adenomas de alto riesgo (AAR), siempre que estén con vigilancia específica; antecedentes personales de adenomas sometidos a seguimiento endoscópico; enfermedad terminal; enfermedad o invalidez grave que contraindiquen el estudio posterior del colon o que requieran un seguimiento específico; y síntomas o signos con valor predictivo alto de CCR.

Se definió la extensión de cobertura poblacional como el \% de la población de Tarjeta Sanitaria que vivía en las Zonas Básicas de Salud en las que se implementó el programa (entendiendo por implementación haber hecho la formación específica de todos los profesionales, médicos y enfermeras, de los Centros de Salud implicados, haber adquirido y distribuido los Hemotest, haber establecido los sistemas de derivación de los positivos, haber instalado el programa informático de evaluación, y haber hecho las adaptaciones necesarias en el hospital de referencia para hacer frente al exceso de colonoscopias). Para realizar el cálculo del número de invitaciones que habría que enviar cada mes, la población total de la zona, dado el carácter bienal del programa, se dividió entre 24.

Prueba para el cribado. Se empleó la determinación de $\mathrm{SOH}$ por método inmunoquímico cualitativo en dos muestras de heces. Es un kit similar al test de embarazo que da un valor cualitativo (sólo «positivo» o «negativo» en lugar de una cifra de hemoglobina cuantificada). El umbral de positividad varía según la marca comercial. Se eligió un umbral de $20 \mu \mathrm{g} / \mathrm{gr}$ de heces por ser el utilizado en la única experiencia previa realizada en España con test inmunoquímico ${ }^{16}$.

Como prueba de confirmación se empleó la colonoscopia óptica (virtual en caso de contraindicación).

Desarrollo del programa. La Consejería de Sanidad organizó la formación previa de los profesionales sanitarios de Atención Primaria, así como la invitación postal (a aquellas personas de la base de datos de Tarjeta Sanitaria que hubieran cumplido año impar el mes anterior entre los 55 y 69 años) inicialmente con una sola carta en la que se señalaba que en caso de aceptar la invitación deberían citarse en la consulta de enfermería de su Centro de Salud. En la carta, también se indicaba que en caso de tener algún familiar de primer grado diagnosticado de cáncer o pólipo colorrectal, deberían de aportar a la cita algún informe médico que lo acreditara y que en caso de encontrarse ya sometidos a algún tipo de vigilancia médica por pólipos o cáncer de colon, no precisaba atender la invitación.

El personal de enfermería de Atención Primaria identificó aquellas personas que presentaban algún tipo de exclusión del programa, y las que tenían algún factor de riesgo de CCR. En el caso de tener algún criterio de exclusión por presentar antecedentes familiares o personales de riesgo incrementado para el CCR, se les dio cita con su médico de familia y fueron derivadas en su mayoría al Servicio de Digestivo. El resto recibió las instrucciones para la recogida de heces (verbal y escrita) dos frascos para la toma de muestras y cita para la lectura del test.

Tras la lectura, aquellos con resultado negativo fueron informados de que serían citados en dos años, y a los positivos se les 
solicitó una colonoscopia en su hospital de referencia, haciéndose entrega de hoja de consentimiento, cuestionario anestésico, volante de analítica (hemograma y coagulación) e instrucciones para la preparación de la colonoscopia.

Las colonoscopias se realizaron con sedación profunda habitualmente con propofol, llevada a cabo por un especialista en anestesiología. Todos los pólipos fueron extirpados si se podía durante la endoscopia para estudio histológico, y si no en una intervención quirúrgica posterior. Los pacientes en los que se detectó un carcinoma invasivo en la colonoscopia fueron remitidos directamente al Servicio de Digestivo para realizarse el estudio de extensión con la menor demora, y posteriormente al Servicio de Cirugía. Los demás fueron informados por su médico de familia, quien recibió el resultado de la endoscopia a través de la historia clínica electrónica, recomendándose en dicho informe el plazo adecuado para el seguimiento siguiente endoscópico en aquellos pacientes que lo precisaron, en función de los hallazgos en esta primera colonoscopia.

Evaluación del programa. Se realizó desde la Dirección General de Salud Pública de acuerdo con los indicadores propuestos por el panel de expertos reunido en Murcia ${ }^{17}$. Cuando se seleccionaron de la base de datos de la Tarjeta Sanitaria las personas a invitar, no se sabía si tenían criterios de exclusión. Por eso se invitó a todas, incluyendo el recordatorio en la carta de que en caso de estar ya sometido a vigilancia médica por pólipos o cáncer de colon no precisaban atender la invitación. En las restantes fue el personal de Atención Primaria el que hizo la anamnesis y decidió si existía motivo de exclusión. Al haber anamnesis previa a la entrega de la muestra de heces, el número de personas que finalmente entregó la muestra fue menor que en otros programas en los cuales no hay este filtro. Es por esto que en Cantabria se definió la tasa de cobertura como el porcentaje de la población con Tarjeta Sanitaria de Cantabria que había recibido al menos una invitación válida (no devuelta por correo) y la tasa de participación como el número de personas que respondiendo a la carta de invitación se presentaron en el Centro de Salud. Los objetivos marcados por el programa para estos dos indicadores fueros $\geq 90 \% \mathrm{y} \geq 30 \%$ respectivamente.

Con el fin de conocer los principales determinantes de la falta de participación en el programa, durante la fase de pilotaje el personal de enfermería de la Sección de Promoción y Educación para la Salud de la Dirección General de Salud Pública, realizó una encuesta telefónica entre una muestra de personas seleccionadas aleatoriamente entre aquellas que cada mes habiendo sido citadas no acudieron al centro de salud. Los resultados de esta encuesta no son objeto de este estudio, es por ello que no haremos referencia a ellos en el apartado de resultados del programa.

En las tablas 1 y 2 se presentan las definiciones y objetivos marcados para cada uno de los indicadores elaborados para evaluar las actividades desarrolladas tanto en atención primaria como especializada cuyos resultados brutos serán presentados en el siguiente apartado.

\section{RESULTADOS}

Se dispuso de datos de Tarjeta Sanitaria de 92.605 personas de 55 a 69 años, siendo la extensión de la cobertura poblacional del $37,8 \%$.

En la tabla 3 se muestran los resultados de las actividades de citación, captación y cribado en Atención Primaria.

Se enviaron 7.771 invitaciones (3.528 enviadas durante el año del pilotaje, más 4.243 mandadas desde enero a marzo de 


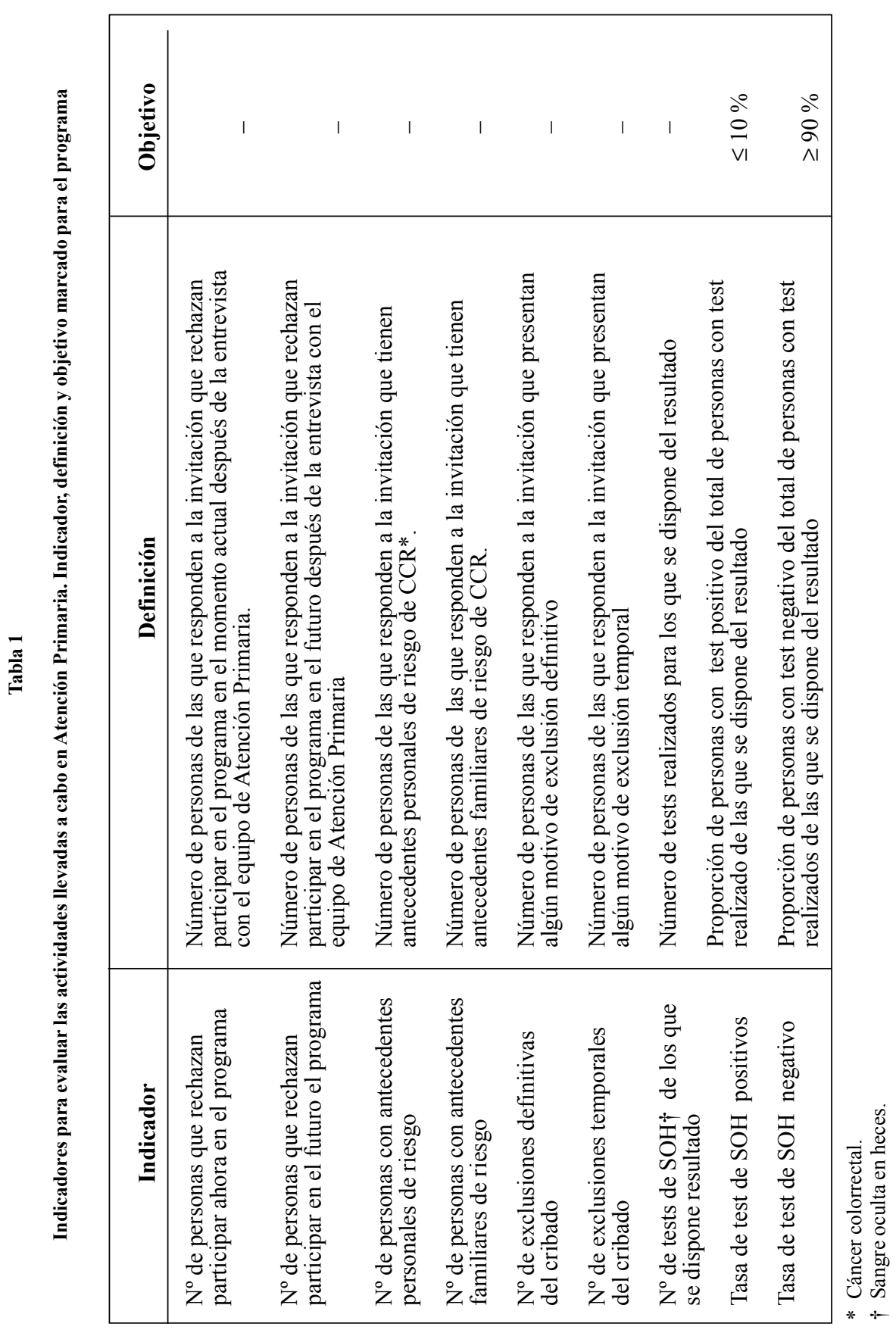




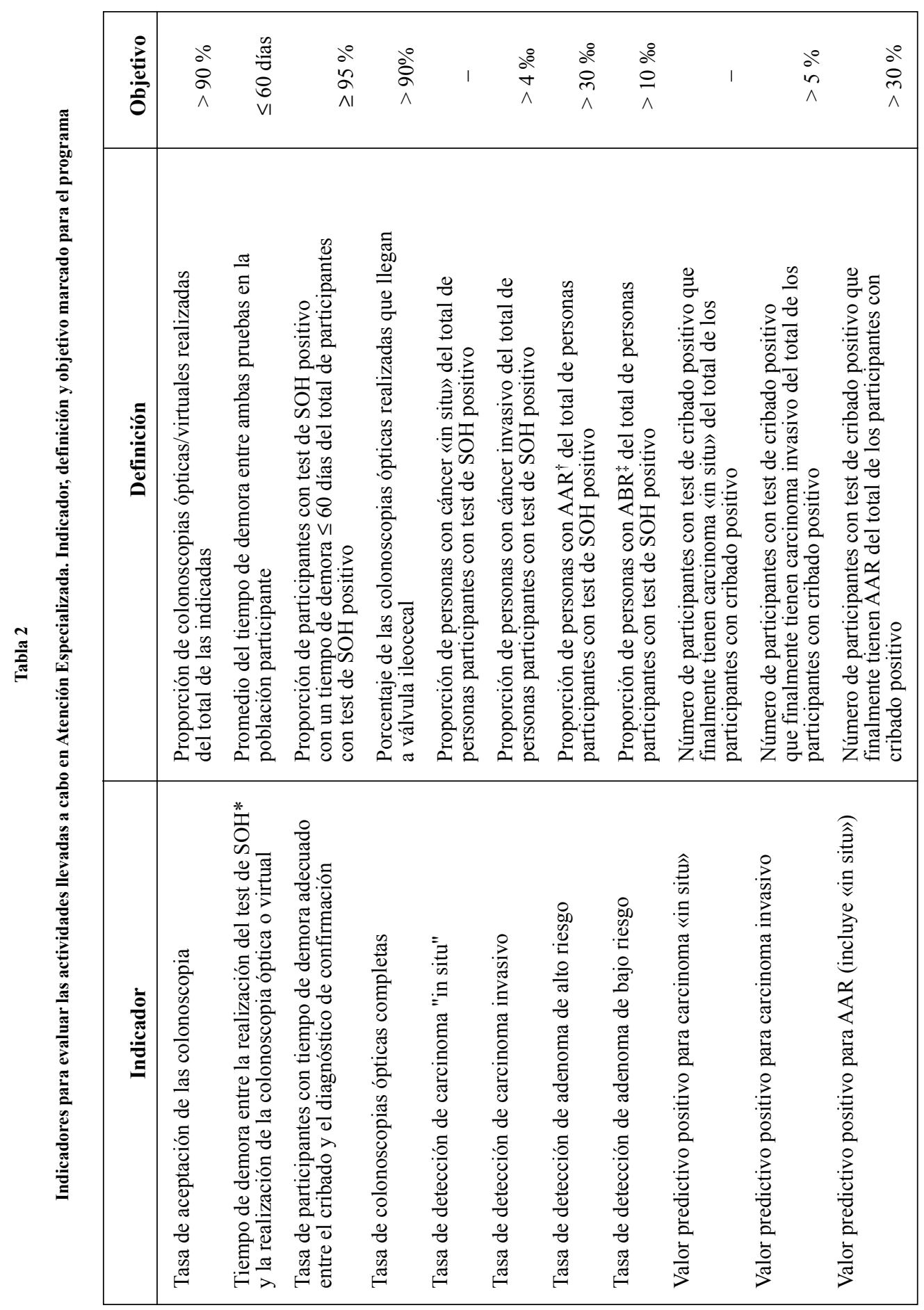




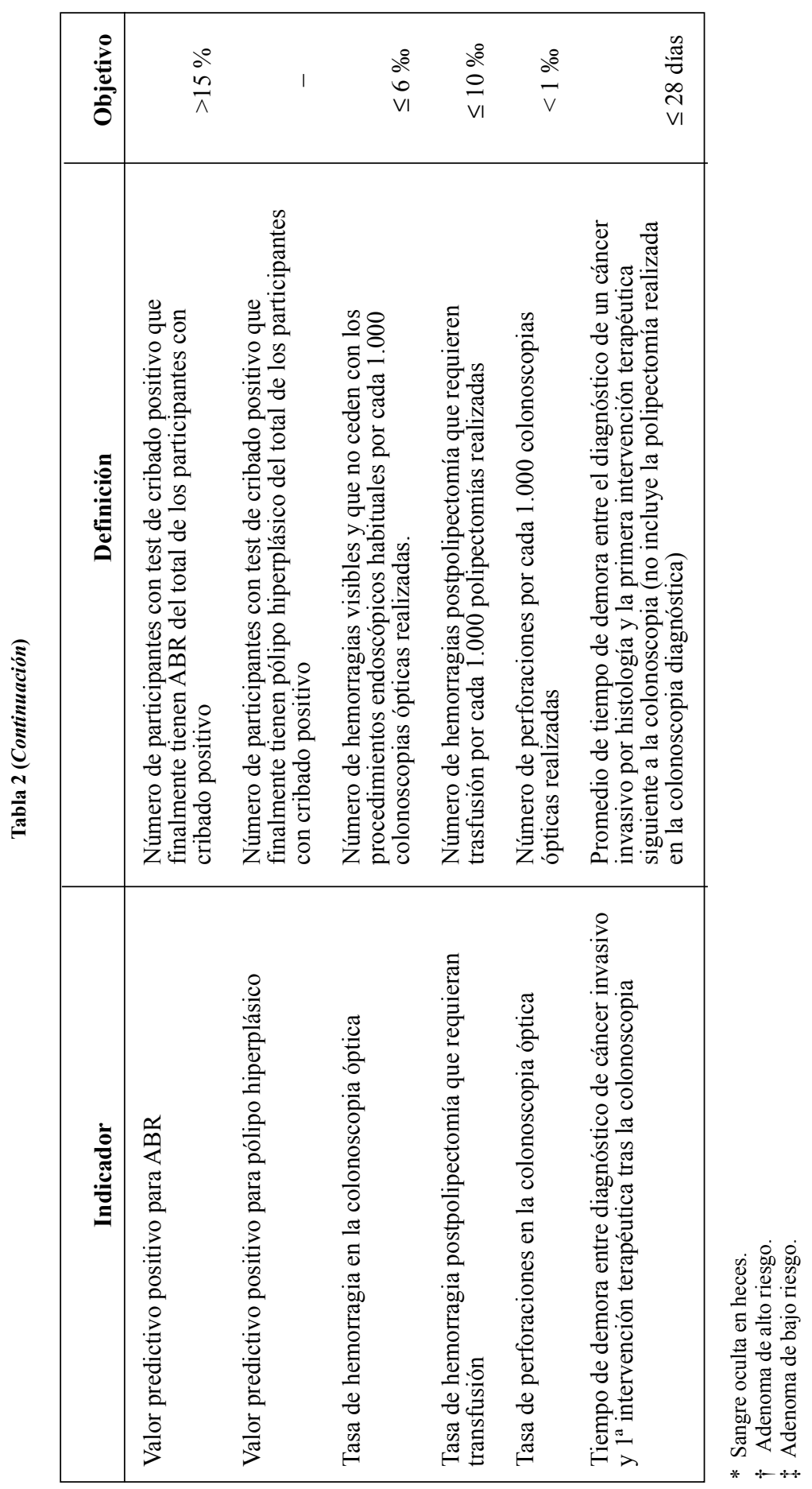

Rev Esp Salud Pública 2010, Vol. 84, N. ${ }^{\circ} 6$ 
Tabla 3

Indicadores en Atención Primaria

\begin{tabular}{|l|c|c|c|}
\hline \multicolumn{1}{|c|}{ Indicador } & $\mathbf{N}$ & $\mathbf{\%}$ & Objetivo \\
\hline Tasa de cobertura & $7.406 / 7.771$ & 95,3 & $\geq 90 \%$ \\
Tasa de participación & $2.384 / 7.406$ & 32,1 & $\geq 30 \%$ \\
Rechazan participar ahora $^{\mid} 39 / 2.384$ & 1,6 & - \\
Rechazan participar futuro $^{\mid} 47 / 2.384$ & 1,9 & - \\
Antecedentes personales de riesgo $^{*}$ & $90 / 2.384$ & 3,7 & - \\
Antecedentes familiares de riesgo $^{\dagger}$ & $206 / 2.384$ & 8,6 & - \\
Exclusión definitiva del cribado $^{*}$ & $41 / 2.384$ & 1,7 & - \\
Exclusión temporal del cribado $^{\S}$ & $99 / 2.384$ & 4,1 & - \\
SOH $^{\|}$de los que se dispone resultado & $1.978 / 2.384$ & 82,9 & - \\
SOH positivo & $143 / 1.978$ & 7,2 & $\leq 10 \%$ \\
SOH negativo & $1.835 / 1.978$ & 92,7 & $\geq 90 \%$ \\
\hline
\end{tabular}

* 17 enfermedad inflamatoria intestinal, 23 cáncer o adenoma previos, y en 50 no consta.

$\dagger$ Sólo 32 casos (el 1,3\%) de suficiente entidad para ser remitidos directamente a la consulta de Digestivo.

$\$ 17$ por estar en seguimiento por antecedentes familiares, 18 por antecedentes personales de CCR o adenoma en seguimiento, 4 por enfermedad terminal o invalidez grave, y 2 por síntomas compatibles con CCR.

$\S 68$ por colonoscopia reciente ya hecha, 12 por fisura anal, y 19 por sangrado hemorroidal.

\| Sangre oculta en heces.

$2.010)$, de las cuales $7.406(95,3 \%)$ fueron válidas (no devueltas por correo). De las 7.406 invitaciones válidas, 2.384 personas acudieron a su Centro de Salud, lo que da una tasa de participación del 32,1\%.

De las 2.384 personas que acudieron al centro de salud, rechazaron participar ahora en el programa $39(1,6 \%)$ y en el futuro 47 $(1,9 \%)$, presentando antecedentes personales o familiares de riesgo $90(3,7 \%)$ y 206 $(8,6 \%)$ personas respectivamente.

Se dispone del resultado de 1.978 test, de los cuales $143(7,2 \%)$ fueron positivos.

En la tabla 4 se presentan los resultados de la actividad realizada en atención especializada. No se incluyen en esta evaluación 9 $(6,2 \%)$ de los 143 pacientes con test positivo por las siguientes causas: 2 rechazaron hacerse la colonoscopia, 4 fueron derivados erróneamente a consulta de digestivo en lugar de endoscopias, 1 se hizo el estudio en otra Comunidad Autónoma, 1 cambió de domicilio y 1 fue remitido a colonoscopia virtual.

La tasa de aceptación de la colonoscopia en el periodo de pilotaje fue del $98 \%$. De las 134 personas derivadas a endoscopia se dispuso del resultado de 91 (67,9\%) siendo el tiempo medio de demora para la realización de la colonoscopia de 45 días.

La tasa de colonoscopias completas fue del 97,8\%. No hubo perforaciones relacionadas con la colonoscopia y la tasa de hemorragias postpolipectomía que requirieron transfusión fue del 28,1 \%o (2 pacientes).

Los principales hallazgos de las colonoscopias fueron los siguientes: 1 carcinoma «in situ», 2 carcinomas invasivos, 43 AAR 
Tabla 4

Indicadores en Atención Especializada

\begin{tabular}{|c|c|c|c|}
\hline Indicador & $\mathbf{N}$ & \% ó \%o & Objetivo \\
\hline Tasa de aceptación de las colonoscopia & & $98 \%$ & $>90 \%$ \\
\hline $\begin{array}{l}\text { Tiempo de demora entre test } \mathrm{SOH}^{*}+ \\
\text { y colonoscopia }\end{array}$ & 45 días & & $\leq 30 \%$ \\
\hline $\begin{array}{l}\text { Tasa de participantes con tiempo de } \\
\text { demora mayor o igual a } 2 \text { meses } \dagger\end{array}$ & $65 / 84$ & $77,3 \%$ & $\geq 95 \%$ \\
\hline Tasa de colonoscopias completas & $89 / 91$ & $97,8 \%$ & $>90 \%$ \\
\hline Colonoscopia normal $\ddagger$ & $4 / 91$ & $4,3 \%$ & \\
\hline \multicolumn{4}{|l|}{ Tasa / 1.000 sometidos a cribado } \\
\hline Carcinoma in situ & $1 / 1.978$ & $0,5 \%$ & - \\
\hline Carcinoma invasivo & $2 / 1.978$ & $1,0 \%$ & $>4 \%$ \\
\hline AAR $\S$ (incluye in situ) & $43 / 1.978$ & $21,7 \%$ & $>30 \%$ \\
\hline $\mathrm{ABR} \|$ & $15 / 1.978$ & $7,5 \%$ & $>10 \%$ \\
\hline \multicolumn{4}{|l|}{ Valor predictivo positivo: } \\
\hline Carcinoma in situ & $1 / 91$ & $1,0 \%$ & - \\
\hline Carcinoma invasivo & $2 / 91$ & $2,1 \%$ & $>5 \%$ \\
\hline AAR (incluye in situ) & $43 / 91$ & $47,2 \%$ & $>30 \%$ \\
\hline ABR & $15 / 91$ & $16,4 \%$ & $>15 \%$ \\
\hline Pólipos hiperplásicos & $25 / 91$ & $27,4 \%$ & - \\
\hline Tasa de hemorragia postcolonoscopia & $3 / 91$ & $32,9 \%$ & $\leq 6 \%$ \\
\hline $\begin{array}{l}\text { Tasa de hemorragia postpolipectomía } \\
\text { que requiera transfusión }\end{array}$ & $2 / 71$ & $28,1 \%$ & $\leq 10 \%$ \\
\hline $\begin{array}{l}\text { Tiempo de demora entre diagnóstico } \\
\text { de cáncer invasivo y } 1^{\mathrm{a}} \text { intervención }{ }^{\mathrm{II}}\end{array}$ & 104 días & & $\leq 28$ días \\
\hline
\end{tabular}

* Sangre oculta en heces.

$\dagger$ Se excluyen 7 pacientes mal remitidos a la consulta de Digestivo en lugar de colonoscopia directamente, o en los que la demora se debe a su patología o tratamientos de base, por lo tanto no atribuible al sistema sanitario.

\$ Se entiende por "colonoscopia normal" la ausencia de cualquier hallazgo responsable del sangrado (divertículos, hemorroides, inflamación de la válvula ileocecal, leiomioma, lipoma, pseudolipomatosis y/o angiodisplasia, etc.). Algunos de estos hallazgos, como la angiodisplasia aunque no se relacionen con el cáncer, también se pueden resolver en la colonoscopia y se evita el sangrado posterior.

$\S$ Adenoma de alto riesgo.

\| Adenoma de bajo riesgo.

II Un paciente con pluripatología que fue remitido a un hospital de referencia distinto del suyo y sometido a múltiples consultas antes de que fuera posible la intervención (161 días); y una paciente remitida a un hospital distinto del de referencia y que también se demoró más (47 días) de lo que habría esperado a través de la derivación normal.

(incluyendo el carcinoma «in situ») y 15 ABR. Los valores predictivos positivos fueron los siguientes: $2,1 \%$ para el carcinoma invasivo, 47,2\% para los AAR (incluyendo el carcinoma «in situ») y $16,4 \%$ para los ABR. 
El tiempo de demora entre el diagnóstico y la $1^{\text {a }}$ intervención terapéutica en los pacientes diagnosticados de cáncer invasivo fue de 104 días.

\section{DISCUSIÓN}

A pesar de estar firmemente recomendado en numerosas estrategias preventivas ${ }^{9-13}$, el cribado del CCR está tardando en introducirse en España probablemente por la escasa percepción social del riesgo, que genera una escasa demanda por parte de la población y, por otra parte, por los recursos sanitarios necesarios para hacer frente a las colonoscopias. En Cantabria se estimó que el programa de cribado aumentaría las colonoscopias que se realizan cada año en toda la red sanitaria, pública y privada, en un $20 \%$, contabilizando sólo las procedentes de la población cribada de riesgo medio y con una participación estimada del $30 \%$.

Ello ha conducido a la paradoja por ejemplo de fomentar la vacunación contra el virus del papiloma y el cribado de cáncer de cérvix siendo éste mucho menos incidente, mientras no se implementaba el cribado del CCR que por sus características tiene una eficiencia mayor. La Estrategia Nacional del Cáncer en España en su más reciente revisión ${ }^{18}$ de 2009 ya recomienda la implementación de programas poblacionales de cribado del CCR con $\mathrm{SOH}$ cada 2 años, cuando la versión anterior ${ }^{19}$ de 2006 sólo recomendaba profundizar en el estudio de las experiencias piloto que estaban entonces desarrollándose.

Cuando Cantabria inició el programa de cribado fue la cuarta Comunidad Autónoma en España en implementarlo (ya lo hacían Murcia, Valencia y Cataluña ${ }^{16,20,21}$ incorporándose poco después el País Vasco y Canarias).

Las peculiaridades que presenta nuestro modelo respecto a los que ya había son básicamente las siguientes:
1) La integración de todo el programa en la red asistencial pública existente.

2) La realización del test de $\mathrm{SOH}$ en el Centro de Salud.

3) La utilización de un test inmunoquímico cualitativo.

La integración en la red asistencial significa no crear una estructura paralela de citación, cribado, derivación y asistencia como se ha hecho por ejemplo en el cáncer de mama. El paciente es atendido por el personal de enfermería de su Centro de Salud, que realiza el test si no existen circunstancias de riesgo, derivándolo a su médico de familia en caso de que existan. Con una participación del 32,1 \% supone una carga asistencial de un paciente cada 1-2 semanas para cada enfermera. Este sistema también permite el registro de las familias de riesgo en Atención Primaria y su adecuada derivación a los servicios asistenciales.

La realización del test en el propio Centro de Salud agiliza el sistema y evita la remisión de muestras a un laboratorio central y posteriormente la devolución de los resultados por correo al usuario o al Centro de Salud. En el mismo momento de la entrega de las muestras el usuario conoce el resultado, $y$, si es positivo, su médico le informa de las implicaciones.

Además de Cantabria, Murcia y País Vasco emplean test inmunoquímicos. La utilización de este test preferentemente al del guayaco (empleado en Cataluña y Valencia) se basa en las experiencias previas que han demostrado su mayor sensibilidad especialmente para adenomas de alto riesgo ${ }^{22,23}$ (prevención primaria), su mayor especificidad al detectar sólo hemoglobina humana procedente de tracto digestivo inferior (no se ve falseado por sangrados altos o por productos de la dieta), su mayor eficiencia, y su mayor comodidad al no necesitar preparación dietética antes de la recogida de la muestra. El 
principal inconveniente es que la tasa de positivos es mayor $(6,5-9,5 \%$ frente a $2-3,4$ $\%)$ lo que genera más derivaciones a colonoscopia. De las tres Comunidades que emplean el test inmunoquímico, únicamente Cantabria emplea el cualitativo. Creemos que este test ofrece las siguientes ventajas:

1) Evita la remisión de heces a un laboratorio. No hay que comprar y mantener equipos, se evitan interrupciones del programa por averías, y no hay que esperar a acumular un determinado número de muestras para analizarlas en serie.

2) La anamnesis por el personal sanitario antes de la recogida garantiza la mejor aplicación de las exclusiones lo que se traduce en menor tasa de falsos positivos.

3) No hay test «inválidos» o con «errores técnicos» (se repiten en el momento con la misma muestra de heces pero con otro kit). No se generan repeticiones.

4) Rapidez: resultado en menos de 5 minutos en lugar de esperar varios días.

5) Inmediatez en la transmisión de resultados: los positivos reciben la información verbal de su médico o enfermera en el momento, no una carta de los administradores del programa varios días después.

6) Economía: 2,1 $€$ determinación.

7) Facilidad y versatilidad en la extensión a nuevas zonas de salud. Sólo precisa realizar la formación del personal y la distribución de los kits. En Cantabria ha permitido extender la implantación del cribado al $82 \%$ de la población en 2 años.

8) Evita el efecto subjetivo del «umbral arbitrario» del test cuantitativo. Este punto hace referencia a que la fijación de un umbral en medicina siempre es arbitraria, lo que vale para la sangre en heces como para cualquier otro parámetro (colesterolemia, tensión arterial, etc.). En la sangre en heces hay que establecer el límite al sangrado intestinal fisiológico, lo que en el test cualitativo es un límite nítido («si»o «no») mientras que en el cuantitativo es más difuso. Un médico que recibe un resultado cuantitativo de sangre en heces puede considerar que su paciente merece un estudio ulterior aunque no alcance el umbral «arbitrariamente» definido por el programa de su comunidad (por ejemplo si la desviación de ese umbral es pequeña) y solicitar más colonoscopias que las previstas, generando una sobreutilización de los recursos y tal vez una mayor yatrogenia. Con el test cualitativo esta duda no se plantea o al menos no es tan explícita.

Respecto a nuestros resultados, resaltamos la participación satisfactoria (32\%) teniendo en cuenta que es con 1 sola carta. En Murcia, por ejemplo, donde en el estudio piloto se enviaron hasta 4 cartas seguidas de una invitación telefónica, la participación tras una carta fue del $30 \%$, ascendiendo al $42 \%$ tras las 4 cartas y la llamada telefónica (Reunión Red de Programas de Cribado de Cáncer Colorrectal. Valencia. Noviembre 2009). En otras Comunidades Autónomas la participación oscila entre el 17,2\% en el caso de Cataluña en la primera vuelta del programa y el 59\% en el País Vasco. En Cantabria, el objetivo inicial es ofrecer el cribado a toda la población más que insistir en lograr una participación alta en una zona pequeña, de ahí que por el momento no se den recitaciones para el programa. Por otra parte el rango de edad seleccionado es de 55-69 años, dado que la tasa de incidencia de CCR en nuestra Comunidad se incrementa significativamente por encima de 55 años. Nuestro primer objetivo es la extensión universal para esta franja de edad y posteriormente se conside- 
rará la ampliación al intervalo de 50-55 años de acuerdo con la estrategia nacional que lo recomienda para el rango de edad de 50 a 69 años ${ }^{18}$

La tasa de positivos y los valores predictivos positivos para cáncer invasivo y AAR con el test inmunoquímico cualitativo han sido similares en nuestra Comunidad respecto a los del test cuantitativo del mismo umbral (VPP para el cáncer invasivo del $2,1 \%$ en Cantabria y del 3,6\% en Murcia, VPP para el AAR del 47,2\% en Cantabria y del 41,2\% en Murcia). (Reunión Red de Programas de Cribado de Cáncer Colorrectal. Valencia. Noviembre 2009).

Tanto la tasa de positivos como el valor predictivo positivo para el carcinoma invasivo, no se han acercado tanto a lo previsto por el programa (objetivos marcados $>4 \%$ y $>$ $5 \%$ respectivamente) como para los adenomas de alto y de bajo riesgo, lo que atribuimos a la pequeña población diana en Cantabria y a la baja prevalencia, que hacen que pequeñas fluctuaciones en el número de casos debidas al azar cambien mucho la tasa.

La calidad de colonoscopias en general ha sido buena, obligándonos a vigilar la tasa de hemorragias postpolipectomía con necesidad de transfusión, que fue superior al objetivo $(28,1 \%$ frente $\mathrm{a} \leq 10 \%$ ), si bien la casuística es aún pequeña y la tasa de hemorragias ha ido disminuyendo a medida que avanzaba el programa (15\%o según el dato más reciente). Como en otros programas, se ha puesto de manifiesto la necesidad de compaginar su implantación con el objetivo de acortar la lista de espera de colonoscopia de los sujetos sintomáticos. En Cantabria se situaba en 5-6 meses antes del programa. La contratación de un endoscopista ha permitido ir logrando ambos objetivos, estando actualmente en 1-2 meses tanto para el cribado como para las personas sintomáticas. Destacamos la alta tasa de patología de cualquier tipo hallada entre las colonoscopias procedentes del cribado $(95,8 \%)$ superior incluso a la encontrada en colonoscopias fuera del cribado ( $83 \%$ en el año 2009) por lo que los procedentes del cribado no se deberían considerar de menor prioridad. Los pacientes que tuvieron patologías no relacionadas con el cáncer también se beneficiaron del programa, pues en la misma colonoscopia se diagnosticó y en muchos casos se resolvió la lesión causante del sangrado. Por lo tanto la selección previa mediante $\mathrm{SOH}$ crea un subgrupo de pacientes asintomáticos pero con una alta prevalencia de lesiones, la mayoría de alto riesgo de cáncer (en nuestra experiencia $47,2 \%$ adenomas de alto riesgo y $2,1 \%$ carcinomas invasivos).

Aunque no es objeto de este estudio, los resultados obtenidos en la encuesta realizada durante el pilotaje para conocer los motivos de rechazo a participar en el programa permitieron determinar que mejorando algunos aspectos como la organización de los servicios o una adecuada educación sanitaria la participación en el programa podría mejorar en un $11 \%$.

En relación a las limitaciones del estudio, hay que señalar que en Cantabria la carta de presentación invita a las personas a autoexcluirse (no ir al Centro de Salud) si ya están en seguimiento colonoscópico, circunstancia que presentaban el 13,1\% según la citada encuesta. Se hizo así para sobrecargar lo menos posible al sistema asistencial. Por la definición de la tasa de participación, cuyo denominador son las invitaciones válidas, definidas como las que no presentan motivo de exclusión, en nuestro caso el denominador estaría reducido en un $13 \%$. Como esta reducción no se ha tomado en cuenta al proceder de una encuesta no diseñada para este fin, al mantener en el denominador todas las invitaciones ha resultado una tasa de participación un $4 \%$ inferior a la que se obtendría aplicando esa corrección.

De cara a próximas vueltas, se estuvo dudando si invitar a aquellas personas en las que constaba que no deseaban participar en 
el programa. Finalmente, se optó por invitarlas ya que este rechazo no se había hecho constar por escrito y había dudas en algunos casos de la certeza de ese rechazo.

A modo de conclusión podemos decir que por su integración en la Atención Primaria, la ausencia de aparataje, fácil uso y rápida realización, el test de detección de $\mathrm{SOH}$ inmunoquímico cualitativo facilita la extensión del cribado, con resultados similares a los obtenidos con los test inmunoquímicos cuantitativos. Para confirmar la disminución de mortalidad descrita en la bibliografía habrá que esperar a disponer estudios de seguimiento a más largo plazo.

\section{AGRADECIMIENTOS}

Agradecemos la colaboración de todo el personal de los Centros de Salud que participaron en el pilotaje (Bajo Asón, Colindres, Laredo y Santoña), de la Gerencia de Atención Primaria Santander-Laredo, y del Servicio de Digestivo del Hospital Universitario Marqués de Valdecilla, sin cuya colaboración y entusiasmo no hubieran sido posibles estos resultados.

\section{BIBLIOGRAFÍA}

1. Parkin MD, Bray F, Ferlay J , Pisani P. Global Cancer Statistics, 2002 CA Cancer J Clin. 2005; 55: 74108.

2. Ferlay J, Autier P, Boniol M, Heanue M, Colombet M, Boyle P. Estimates of the cancer incidence and mortality in Europe in 2006. Ann Oncol. 2007; 18: 581-592.

3. Domenech A, Pérez-Gómez B, Aragonés N, Pollán M, López-Abente G. La situación del cáncer en España. 1975-2006. Madrid: Centro Nacional de Epidemiología; 2009.

4. Mandel JS, Bond JH, Church TR, Snover DC, Bradley GM, Schuman LM «et al. « Reducing mortality from colorectal cancer by screening for fecal occult blood. Minnesota Colon Cancer Control Study. N Engl J Med. 1993; 328:1365-1371.
5. Hardcastle JD, Chamberlain JO, Robinson $\mathrm{MH}$, Moss SM, Amar SS, Balfour TW, «et al. «. Randomised controlled trial of faecal-occult-blood screening for colorectal cancer. Lancet. 1996; 348:14721477.

6. Kronborg O, Fenger C, Olsen J, Jorgensen OD, Sondergaard $\mathrm{O}$. Randomised study of screening for colorectal cancer with faecal-occult-blood test. Lancet. 1996; 348:1467-1471.

7. Scholefield JH, Moss S, Sufi F, Mangham CM, Hardcastle JD. Effect of faecal occult blood screening on mortality from colorectal cancer: results from a randomised controlled trial. Gut. 2002; 50: 840-844.

8. Mandel JS, Church TR, Bond JH, Ederer F, Geisser MS, Mongin SJ, «et al. «. The effect of fecal occultblood screening on the incidence of colorectal cancer. N Engl J Med. 2000; 343: 1603-1607.

9. Canadian Task Force on Preventive Health Care. Colorectal cancer screening. Recommendation statement from the Canadian Task Force on Preventive Health Care. JAMC. 2001; 165 (2): 206-08.

10. Comisión de las Comunidades Europeas. Propuesta de Recomendación del Consejo sobre cribado del cáncer. Bruselas; 2003. 230 final 2003/0093 (CNS).

11. Boyle P, Autier P, Bartelink H, Baselga J, Boffetta P, Burn J «et al. «. European Code Against Cancer and scientific justification: third version. Ann Oncol. 2003; 14: 973-1005.

12. U. S. Preventive Services Task Force. Screening for Colorectal Cancer. Ann Intern Med. 2008; 149:627-637.

13. Grupo de trabajo de la guía de práctica clínica de prevención del cáncer colorrectal. Actualización 2009. Guía de práctica clínica. Barcelona: Asociación Española de Gastroenterología, Sociedad Española de Medicina de Familia y Comunitaria, y Centro Cochrane Iberoamericano; 2009. Programa de Elaboración de Guías de Práctica Clínica en Enfermedades Digestivas, desde la Atención Primaria a la Especializada; 4.

14. Instituto Nacional de Estadística. Avance del Padrón a 1 de enero de 2010. Datos provisionales. [citado 5 mayo 2010]. www.ine.es/jaxi/tabla.do? path $=/$ t20/e245/p04/provi/10/\&file $=0$ ccaa001.PX $\&$ type $=$ pcaxis $\& \mathrm{~L}=0$

15. Programa de Detección Precoz del Cáncer Colorrectal en Cantabria. Gobierno de Cantabria. Con- 
sejería de Sanidad. Dirección General de Salud Pública. Santander: Consejería de Sanidad; 2008.

16. Pérez-Riquelme F, Cruzado Quevedo J, Carballo Álvarez L, Torrella Cortés EA., Lázaro Agustín MD, Cavas Martínez MC «et al.» Prevención del cáncer de colon y recto en la. Prevención del cáncer de colon y recto en la Región de Murcia. Proyecto Piloto. En: Pérez-Riquelme F, Cruzado Quevedo J, Gutierrez-García JJ. Editores. La prevención del cáncer de colon y recto en la Región de Murcia, Murcia: Consejería de Sanidad de la Región de Murcia; 2008. Serie Informes No: 50. p.

17. Recomendaciones del Panel Nacional de Expertos para planificación y puesta en marcha de programas organizados de base demográfica para la prevención del Cáncer de Colon y Recto. Murcia: Consejería de Sanidad y Consumo de Murcia. Dirección General de Salud Pública. Servicio de Promoción y Educación para la Salud; 2007.

18. Estrategia en Cáncer del Sistema Nacional de Salud. Madrid: Ministerio de Sanidad y Políticas sociales; 2009.
19. Estrategia en Cáncer del Sistema Nacional de Salud. Madrid: Ministerio de Sanidad y Consumo; 2006.

20. Programa de Prevención de Cáncer Colorrectal en la Comunitat Valenciana. Conselleria de Sanitat. Valencia: Generalitat Valenciana; 2009.

21. Peris M, Espinas J, Muñoz L, Navarro M, Binefa G, Borrás $\mathrm{M}$ et al. Lessons learnt from a populationbased pilot programme for colorectal cancer screening in Catalonia (Spain). J Med Screen. 2007; 14:81-86.

22. Guittet L, Bouvier V, Mariotte N, Vallee JP, Arsene $\mathrm{D}$, Boutreux $\mathrm{S}$ et al. Comparison of a guaiac based and an immunochemical faecal occult blood test in screening for colorectal cancer in a general average risk population. Gut. 2007; 56: 210-214.

23. Dancourt V, Lejeunea C, Lepage C, Marie Gailliardb MC, Menya B, Faivrea J. Immunochemical faecal occult blood tests are superior to guaiacbased tests for the detection of colorectal neoplasms. Eur J Cancer. 2008; 44: 22 54-2258. 